\title{
Failure and volume fraction dependent mechanical properties of composite sensors and actuators
}

\author{
C R Bowen ${ }^{1 *}$, A C Dent ${ }^{1}$, L J Nelson ${ }^{1}$, R Stevens ${ }^{1}$, M G Cain ${ }^{2}$, and M Stewart ${ }^{2}$ \\ ${ }^{1}$ Department of Mechanical Engineering, University of Bath, Bath, UK \\ ${ }^{2}$ Functional Materials Group, National Physical Laboratory, Teddington, UK \\ The manuscript was received on 6 December 2005 and was accepted after revision for publication on 8 February 2006.
}

DOI: 10.1243/09544062JMES255

\begin{abstract}
Composite actuators and sensors manufactured by combining a ferroelectric ceramic such as lead zirconate titanate and a passive phase such as a polymer are used in a variety of applications including SONAR, vibration damping, change of structural shape (morphing), and structural health monitoring. The composite route provides specific advantages, including tailored piezoelectric response, high strain, a degree of flexibility, and increased damage tolerance compared with conventional dense monolithic ceramic materials. For piezoelectric fibre composites, where fine-scale brittle ceramic fibres of 40-800 $\mu \mathrm{m}$ diameter are introduced into a ductile polymer matrix, the composite strength and failure mechanism ultimately depend on the mechanical properties of each phase and their volume fraction. This article examines the mechanical properties of piezoelectric fibres and the matrix phase and discusses the possible influence of fibre volume fraction on mechanical properties and failure mechanism of the composite. The data are of particular use in determining the failure stress, failure strain, and failure mechanism of composite actuators and sensors subjected to high levels of stress, for example, in applications where such devices are embedded into host structures.
\end{abstract}

Keywords: fracture, composite, design, sensors, actuators, piezoelectric, active fibre composite

\section{INTRODUCTION}

Piezoelectric materials, which develop an electrical charge when subjected to a force, and a strain when subjected to an electric field, are used in a variety of sensors and actuator applications. The most commonly used piezoelectric ceramic is lead zirconate titanate, $\mathrm{Pb}(\mathrm{Zr}, \mathrm{Ti}) \mathrm{O}_{3}(\mathrm{PZT})$. In the last few years, a variety of micron-scale PZT fibres (40$800 \mu \mathrm{m}$ in diameter) have been manufactured by various methods including sol-gel, extrusion, and viscous suspension spinning, some of which are now commercially available [1-4]. The applications for these fine-scale fibres include 1-3 composites, which are aligned fibres embedded in a polymer matrix for medical transducer and SONAR applications $[\mathbf{5}, \mathbf{6}]$, and active fibre composites (AFCs) that have a variety of potential benefits over

*Corresponding author: Materials Research Centre, Department of Mechanical Engineering, University of Bath, Bath BA2 7AY, UK.email:c.r.brown@bath.ac.uk conventional piezoelectric sensing and actuating devices $[\mathbf{7}, \mathbf{8}]$. AFCs were developed by the Active Materials and Structures Laboratory and patented in 1994 [8]. Since their initial development, advances have been made in many areas including fibre manufacture, matrix materials, electrode design, manufacturing techniques, and composite modelling $[9,10]$. A typical AFC configuration is shown in Fig. 1 and comprises a monolayer of uniaxially aligned piezoelectric fibres embedded in a polymer matrix between two interdigitated electrodes (IDEs) through which the driving voltage and associated electric field are applied. This configuration combines IDEs and a composite architecture to overcome the limitations associated with monolithic structural actuators. The advantage of the IDE configuration is that the electric field is applied in the direction of actuation and the fibre direction, which results in the larger $d_{33}$ piezoelectric coefficient being utilized (the smaller $d_{31}$ coefficient used in monolithic actuators is typically $\sim 0.5 d_{33}$ ). The introduction of fine-scale fibres into a polymer matrix also 


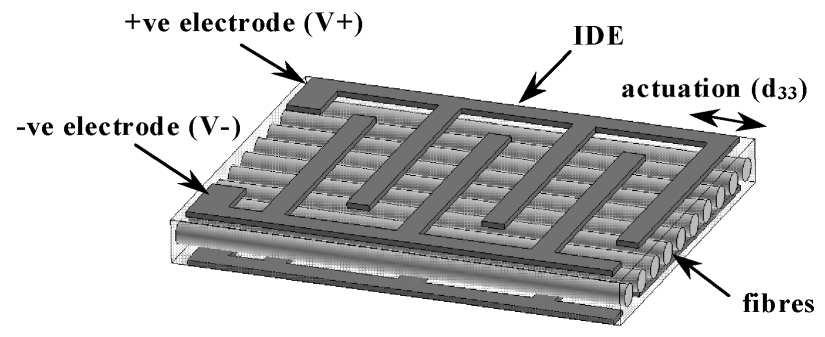

Fig. 1 AFC construction using micron-scale fibres and an IDE pattern

provides the composite with a degree of flexibility and is able to conform to the shape of irregular structures $[\mathbf{8}, \mathbf{1 1}, \mathbf{1 2}]$, a property that has attracted significant interest in research into embedded actuators for change of structural shape (morphing). It has also been suggested that the combination of IDEs and ceramic fibres offers an enhanced toughness and damage tolerance, because fracture of individual fibres does not lead to ultimate failure of the device. As a result of these potential advantages, applications for AFCs include shape control, structural health monitoring, and vibration control [12-14].

If AFCs are to be attached or embedded in engineering structures, knowledge of the mechanical properties of the AFC is essential to prevent failure and loss of strain or sensing capability. Failed or fractured AFCs may also act as a point of weakness in the host structure. This article determines the mechanical properties, in particular, the tensile strength and failure strain, of piezoelectric fibre-based composites as a function of fibre fraction. The change in failure mechanism (related to the 'minimum' volume fraction) and 'critical' volume fraction (the volume fraction in which any strengthening effect of the fibres is observed) is also determined and discussed.

\section{MECHANICAL PROPERTIES OF ACTIVE COMPOSITES}

\subsection{Failure of active composites}

The influence of composite architecture on composite tensile strength and fracture mechanism is well known and has been studied extensively for structural composites consisting of brittle fibres in a ductile matrix $[\mathbf{1 5}, \mathbf{1 6}]$. However, it is surprising to note that the approach has not been applied to determine the important parameters for AFCs, as the approach is equally valid for brittle piezoelectric fibres in a ductile polymer matrix.

Figure 2 shows a cross-section of an AFC [17], showing the piezoelectric fibres, polymer matrix, and electrodes. It is assumed that the composite

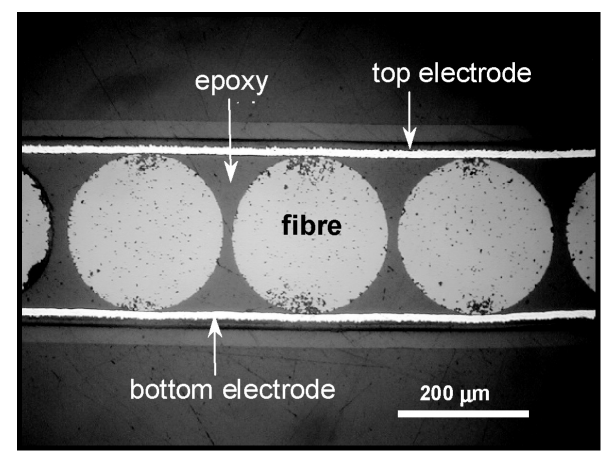

Fig. 2 Cross-section of an AFC, showing PZT fibre, epoxy matrix, and electrodes [17]

consists of long fibres, aligned in the loading direction. The fibres and matrix are considered to be perfectly bonded and have equal levels of deformation (i.e. the strain in the fibres, matrix, and composite are identical). The influence of the mechanical properties of the electrodes, which are orientated perpendicular to the fibre, is ignored. As the system consists of relatively brittle ceramic fibres in a ductile polymer matrix, the failure strain of the matrix $\left(\varepsilon_{\mathrm{m}}^{*}\right)$ is considered greater than that of the fibres $\left(\varepsilon_{\mathrm{f}}^{*}\right)$.

If a tensile mechanical load is applied to the composite along the fibre direction, the composite strain $\left(\varepsilon_{\mathrm{m}}^{*}\right)$ will increase, and the composite modulus ( $\left.E_{\text {composite }}\right)$ is given as

$$
E_{\text {composite }}=V_{\mathrm{f}} E_{\mathrm{PZT}}+\left(1-V_{\mathrm{f}}\right) E_{\text {matrix }}
$$

where $E_{\mathrm{PZT}}$ and $E_{\text {matrix }}$ are the tensile moduli of the PZT fibres and epoxy matrix, respectively, and $V_{\mathrm{f}}$ is the fibre volume fraction. Equation (1) requires both components to have similar Poisson's ratios, which is valid because PZT-5A $v \sim 0.38$ and epoxy $v \sim 0.37[18,19]$.

As the load is increased, the composite strain will reach the failure strain $\left(\varepsilon_{\mathrm{f}}^{*}\right)$ of the brittle fibres, which will be the first component to fail (Fig. 3(a)). At this point, either of two possible failure regimes exists (regime I or regime II), depending on whether

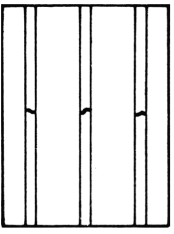

(a) $\stackrel{*}{\varepsilon_{c}}=\stackrel{*}{\varepsilon}$

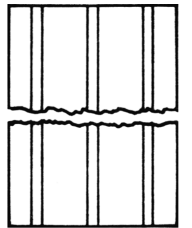

(b) Regime I, $V_{f}>V_{\text {min }}$

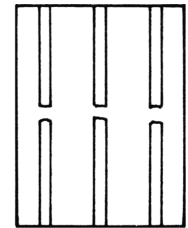

(c) Regime II, $V_{f}<V_{\min }$

Fig. 3 Diagram of possible failure mechanisms for composite system with $\left(\varepsilon_{\mathrm{f}}^{*}<\varepsilon_{\mathrm{m}}^{*}\right)$. The fibres are considered to have uniform strength 
the fibre volume fraction is above or below a minimum threshold value $\left(V_{\mathrm{min}}\right)$.

\subsection{Regime I $\left(V_{\min }<V_{\mathrm{f}}<1\right)$}

If $V_{\mathrm{f}}$ is above $V_{\min }$, brittle failure of the fibres at $\varepsilon_{\mathrm{f}}^{*}$ leads to failure of the entire composite, as the polymer matrix is unable to support the additional load that is transferred to the matrix after fibre failure (Fig. 3(b)). The strength of the composite in this range of fibre volume fractions is described [15] as

$$
\sigma_{\mathrm{C}}^{*}=V_{\mathrm{f}} \sigma_{\mathrm{f}}^{*}+\left(1-V_{\mathrm{f}}\right) \sigma_{\mathrm{m}}^{\prime}
$$

where $\sigma_{\mathrm{c}}^{*}$ is the composite failure stress, $\sigma_{\mathrm{f}}^{*}$ is the fibre failure stress, and $\sigma_{\mathrm{m}}^{\prime}$ the stress carried by the polymer matrix at the fibre failure strain. In the $V_{\mathrm{f}}>V_{\text {min }}$ regime, failure of the fibres leads to complete composite failure and $\varepsilon_{\mathrm{c}}^{*}=\varepsilon_{\mathrm{f}}^{*}$. Composite failure strength is dominated by the fibre properties in this regime.

\subsection{Regime II $\left(0<V_{\mathrm{f}}<V_{\text {min }}\right)$}

If $V_{\mathrm{f}}$ is below $V_{\text {min }}$, the fibre fraction is sufficiently low that the polymer matrix is able to carry the applied load after the fracture of the piezoelectric fibres (Fig. 3(c)). Failure of the piezoelectric fibres at $\varepsilon_{\mathrm{f}}^{*}$ does not lead to composite failure; it merely increases the stress and strain in the polymer matrix. The failed fibres, which now carry no load, can be regarded as holes in the form of cylinders in the polymer matrix, and the tensile strength of the composite is described as [15]

$$
\sigma_{\mathrm{C}}^{*}=\left(1-V_{\mathrm{f}}\right) \sigma_{\mathrm{m}}^{*}
$$

where $\sigma_{\mathrm{m}}^{*}$ is the matrix failure stress. From this, it is assumed that the only effect of the fibres is to decrease the area of the matrix that can be loaded. However, depending on the toughness of the matrix, it is possible that the discontinuities introduced by the broken fibres can cause stress concentrations, further weakening the matrix.

In the $V_{\mathrm{f}}<V_{\min }$ regime, composite failure occurs when $\varepsilon_{\mathrm{c}}^{*}=\varepsilon_{\mathrm{m}}^{*}$ resulting in a more ductile, less catastrophic failure mode. As it is possible that a PZT fibre composite with IDEs can still actuate after individual fibre failures, this is potentially the most appropriate range of fibre volume fractions for the AFCs. Although this regime provides improved toughness, the composite tensile strength will actually decrease with increasing fibre volume fraction within the range $0<V_{\mathrm{f}}<V_{\min }$. Composite failure strength is dominated by the matrix properties in this regime.

\subsection{Minimum and critical volume fractions}

The minimum volume fraction $\left(V_{\mathrm{min}}\right)$ determines the transition from each regime and failure mechanism and corresponds to the lowest composite strength. From equations (2) and (3) [15]

$$
V_{\min }=\frac{\sigma_{\mathrm{m}}^{*}-\sigma_{\mathrm{m}}^{\prime}}{\sigma_{\mathrm{f}}^{*}+\sigma_{\mathrm{m}}^{*}-\sigma_{\mathrm{m}}^{\prime}}
$$

The critical volume fraction ( $\left.V_{\text {crit }}\right)$ for the composite system is the fibre fraction necessary to ensure that the composite strength is at least greater than that of the matrix $\left(\sigma_{\mathrm{c}}^{*}>\sigma_{\mathrm{m}}^{*}\right)$. Rearranging Equation 2 , with the condition that $V_{\mathrm{f}}=V_{\text {crit }}$ when $\sigma_{\mathrm{c}}^{*}=\sigma_{\mathrm{m}}^{*}$ [15], gives

$$
V_{\text {crit }}=\frac{\sigma_{\mathrm{m}}^{*}-\sigma_{\mathrm{m}}^{\prime}}{\sigma_{\mathrm{f}}^{*}-\sigma_{\mathrm{m}}^{\prime}}
$$

For brittle fibres, such as PZT, potential errors in equations (4) and (5) are associated with the inherent statistical distribution of strength [15].

\section{EXPERIMENTAL}

To determine the necessary composite mechanical properties as a function of fibre volume fraction, the relevant properties $\left(\varepsilon_{\mathrm{f}}^{*}, \sigma_{\mathrm{f}}^{*}, \sigma_{\mathrm{m}}^{*}\right.$, and $\left.\sigma_{\mathrm{m}}^{\prime}\right)$ of the component phases were determined by tensiletesting individual fibres for two types of PZT (PZT-5H and PZT-5A) and fabricating tensile-test specimens of the epoxy polymer matrix.

\subsection{Matrix tensile strength}

The matrix was a low viscosity epoxy resin (Struers Specifix-40) that was cast into tensile specimens and tested in accordance with EN ISO 527-2:1996 [20]. Tensile testing was performed using an Instron 1195 with a $500 \mathrm{~kg}$ load cell and self-centring grips. Displacements were measured using a $50 \mathrm{~mm}$ extensometer and data captured with 'Spider-8' hardware connected to a PC. Testing was performed with a crosshead speed of $5 \mathrm{~mm} / \mathrm{min}$ and data sampled at a rate of $50 \mathrm{~Hz}$.

\subsection{Single-fibre tensile strength}

\subsubsection{Methods}

The fibres examined in this study were PZT-5H and PZT-5A, commonly used sensor and actuator materials and available in fibre form. The chosen fibres were produced by the Alceru method, which were selected because of their commercial 
availability in large quantities. To minimize errors in cross-sectional area measurement, relatively large $(800 \mu \mathrm{m})$ fibres with less variability in diameter and easier to handle were chosen. The use of larger diameter fibres also minimizes errors associated with specimen clamping and measurement of failure loads. In total, 75 unpoled fibres of PZT-5A and 40 fibres of PZT-5H were tested to allow Weibull statistical analysis of the results.

The fibres were tested following the single-fibre tensile test described in European prestandard ENV 1007-4:1994 [21]. A test coupon was used to support the single fibre while preparing and loading the specimen for testing. Once clamped for tensile testing, the coupon was cut so that the entire applied load is transferred to the fibre. The coupon design was initially based on the prestandard 1007-4, currently, on a draft with limited detail on the coupon geometry and use. The end tabs were extended so that they could be securely clamped into the testing machine, as it was found directly clamping the fibres could induce premature failure. The prototype design suffered from a lack of mechanical flexibility, leading to the fracture of the brittle fibres during loading for testing. This was overcome by adding creases to the end tabs, so that the coupon could flex and the fibre was not unduly compressed. Additional marks were printed onto the coupon to facilitate accurate alignment of the test rig, which helps to reduce off-axis loading and increase the accuracy of the measurement of failure load. Figure 4 shows a diagram of the final coupon developed.

A cyanoacrylate and an epoxy were evaluated as potential adhesives to bond the fibre to the test coupons. It was found that owing to fibre wetting with the cyanoacrylate, stress concentrations were higher, which reduced the apparent measured tensile strength of the samples. As a result, epoxy was selected to bond the fibre and test coupon. Tensile testing was performed using an Instron 1122 with a $500 \mathrm{~kg}$ load cell. Specimens were clamped using lightweight pneumatic grips and load applied at a constant crosshead rate of $5 \mathrm{~mm} / \mathrm{min}$. This relatively high loading speed was selected to reduce the ratedependent plastic contribution of ferroelastic switching. Typical failure loads were $\sim 20 \mathrm{~N}$ for both PZT-5A and PZT-5H fibres.

In calculating the failure stress, the variability in fibre cross-section was a considerable source of inaccuracy. Errors due were minimized by measuring the fibre diameter close to the fracture surface. Fibres were inspected with scanning electron microscopy, using the backscattered electron imaging (BEI) to allow automated image analysis of the fibre. Digital micrographs were analysed with the open-source software 'ImageJ' (version 1.34f) [22] to measure the fibre area directly near the fracture site and calculate

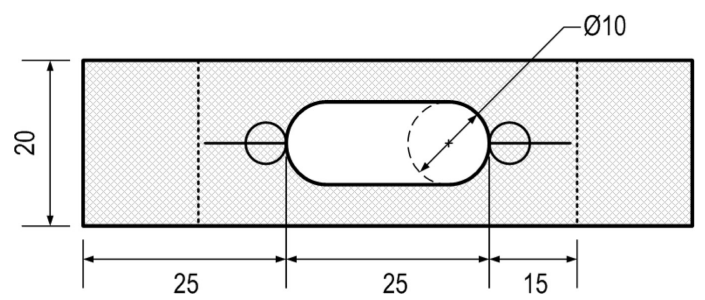

Fig. 4 Tensile test coupon (dimensions in $\mathrm{mm}$ ). A single fibre is located in the centre using the alignment marks. The epoxy is applied within the marked circles to ensure repeatability. The ends of the coupons are gripped above the fold marks (dotted line), which provide mechanical flexibility while loading the specimen

the failure stress for the individual fibre. Although time consuming, direct measurement of the fibre cross-sectional area led to significant improvement in the accuracy of the fracture strength.

\subsubsection{Single fibre results}

Weibull analysis has been widely employed to describe the statistical distribution of failure strengths in ceramic materials [23]. For a volume of ceramic $V$ under a tensile stress $\sigma$, the probability of survival is

$$
P_{\mathrm{s}}(V)=\exp \left\lfloor-\left(\frac{\sigma}{\sigma_{0}}\right)^{m} \cdot \frac{V}{V_{0}}\right\rfloor
$$

where $\sigma_{0}, V_{0}$, and $m$ are constants of the material sample. Although the failure probability is a function of volume, it may be assumed that fibres sampled had constant geometry, and the influence, $V=V_{0}$, may be ignored. The scale parameter $\sigma_{0}$ is effectively an average strength (MPa) and the shape parameter $m$ is inversely proportional to the distribution of the strength values. High-scale and shape parameters would describe the distribution for high strength and homogeneous fibres, whereas low-scale and shape parameters would indicate weak fibres with the presence of variable flaws.

By plotting $\ln \left[-\ln \left(1-P_{\mathrm{f}}\right)\right]$ against the failure strength, $\ln (\sigma)$, for each fibre, it is possible to perform a linear regression from which the scale and shape parameters may be determined. Results of this analysis for PZT-5A and PZT-5H fibres can be seen in Figs 5 and 6, respectively. For both the materials examined, high-quality linear Weibull plots were produced. PZT-5A strength parameters were found to be $\sigma_{0}=39.5 \mathrm{MPa}$ and $m=6.55$, and for PZT-5H, $\sigma_{0}=33.8 \mathrm{MPa}$ and $m=7.22$. This indicates that PZT-5A fibres are slightly stronger than PZT-5H, but exhibit a slightly higher variability in strength. 


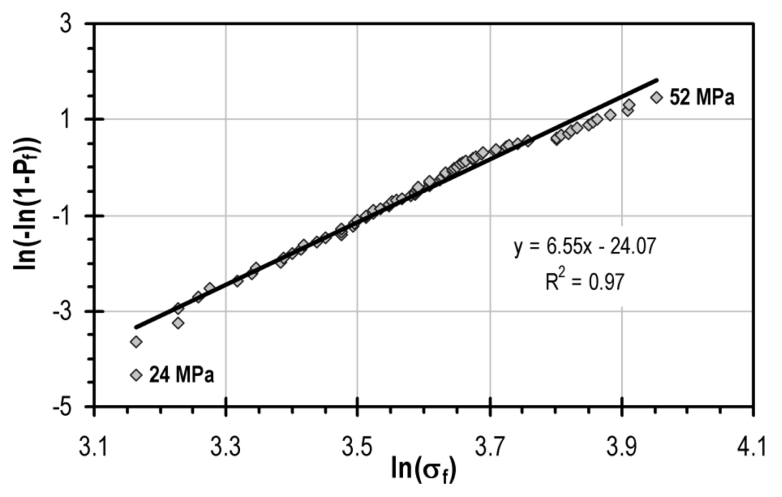

Fig. 5 Weibull plot for PZT-5A fibres with $800 \mu \mathrm{m}$ diameter, produced by the Alceru method. PZT-5A strength parameters are $\sigma_{0}=39.5 \mathrm{MPa}$ and $m=6.55$

The values of $\sigma_{0}$ and $m$ reported by Kornmann and Huber [24] for PZT-5A varied from $\sim 50$ to $64 \mathrm{MPa}$ and from $\sim 4$ to 12 , respectively. The shape parameters are in good agreement with the measured results, suggesting a similar distribution of failure strengths, and hence, a similar distribution of flaws in the samples. However, the tensile strengths reported are higher than those observed in this work. Although this might be attributed to differences in the material, it is likely to originate from differences in testing methodology. Kornmann and Huber [24] loaded the specimens with a low crosshead speed of $0.3 \mathrm{~mm} / \mathrm{min}$, as opposed to $5 \mathrm{~mm} /$ min used in this work. The use of more gradual loading will allow the fibres to deform through ferroelastic switching and may also allow the bonding adhesive and sample coupon to creep. Such high values for strength may overestimate the performance of the fibres in service, where sudden impact loads are most likely to be encountered. Tensile strengths reported for bulk materials [19] suggest a

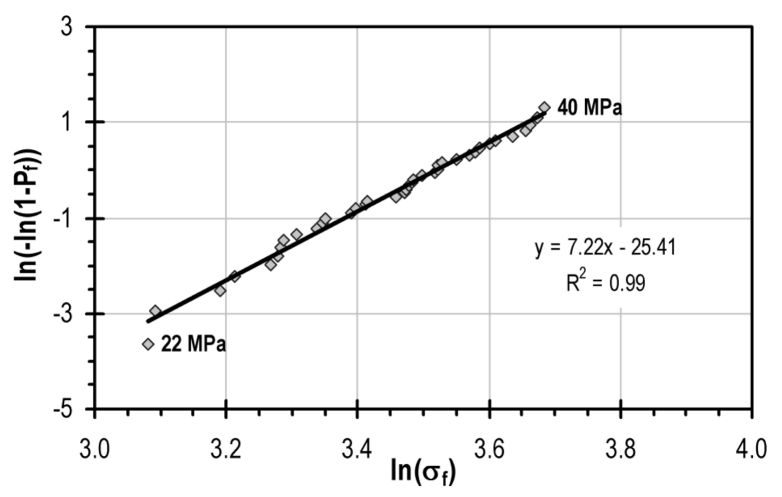

Fig. 6 Weibull plot for PZT-5H fibres with $800 \mu \mathrm{m}$ diameter, produced by the Alceru method. PZT-5H strength parameters are $\sigma_{0}=33.8 \mathrm{MPa}$ and $m=7.22$

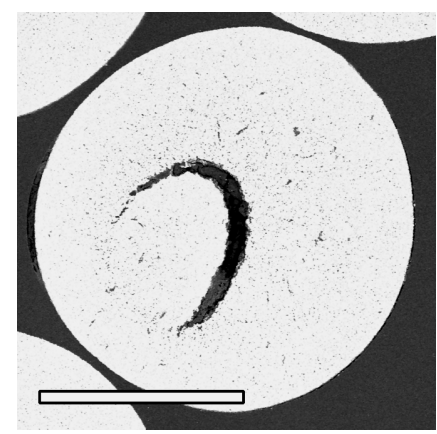

Fig. 7 PZT-5H fibre cross-section close to the point of fracture, with macro-porosity evident and surrounding micro-porosity revealed by the BEI. However, typically, only micro-porosity was observed in the fibres. Scale bar: $400 \mu \mathrm{m}$

'static' strength of $\sim 76 \mathrm{MPa}$ and a 'dynamic' strength of $\sim 28 \mathrm{MPa}$, which support the current findings.

Fibre cross-sections were inspected near the point of fracture to allow accurate measurement of area and hence accurate estimation of stress; the examination also allowed sources of failure to be identified. In one PZT-5H fibre, extensive macro-porosity was seen and that had correspondingly the lowest strength $\sim 22 \mathrm{MPa}$ (Fig. 7). However, typically, only micro-porosity was observed in the fibres near the point of fracture. No noticeable differences were found in the pore structures of the two fibre materials, which is consistent with the similar values for shape factor (7.2 and 6.6) recorded. It is likely that the difference in strength of the two materials results from the different compositions and variations in processing, with the 'harder' PZT5A displaying greater mechanical strength.

\section{COMPOSITE STRENGTH PREDICTIONS}

\subsection{Fibre properties $\left(\varepsilon_{\mathrm{f}}^{*}\right.$ and $\left.\sigma_{\mathrm{f}}^{*}\right)$}

The failure strength of the PZT-5H fibres $\left(\sigma_{\mathrm{f}}^{*}\right)$, determined from the scale parameter $\left(\sigma_{0}\right)$ of the Weibull analysis, was $34 \mathrm{MPa}$. The elastic modulus for the unpoled PZT-5H was $66 \mathrm{GPa}$, estimated from the average of the open- and closed-circuit stiffness of the poled material. Assuming linear elastic behaviour of the fibre up to the failure stress, the fibre failure strain $\left(\varepsilon_{\mathrm{f}}^{*}\right)$ is $\sim 0.05$ per cent. This assumption neglects additional strain due to domain wall motion under the applied stress (ferroelastic behaviour), which is reasonable during rapid loading.

The failure strength of the PZT-5A fibres $\left(\sigma_{\mathrm{f}}^{*}\right)$ was determined to be $40 \mathrm{MPa}$ from the Weibull scale parameter $\left(\sigma_{0}\right)$. The elastic modulus for the unpoled PZT-5A material was calculated as $65 \mathrm{GPa}$, estimated from the open- and closed-circuit stiffness of the 
poled material. Assuming linear elastic behaviour of the fibre up to the failure stress, the fibre failure strain $\left(\varepsilon_{\mathrm{f}}^{*}\right)$ is $\sim 0.06$ per cent.

\subsection{Matrix properties ( $\sigma_{\mathrm{m}}^{*}$ and $\left.\sigma_{\mathrm{m}}^{\prime}\right)$}

The epoxy tensile strength $\left(\sigma_{\mathrm{m}}^{*}\right)$ was determined to be $57 \mathrm{MPa}$ from the average strength of 20 specimens, with a standard deviation of $\pm 2 \mathrm{MPa}$. Assuming linear elastic behaviour and taking the measured elastic modulus of the matrix ( $3 \mathrm{GPa}$ ), the stress levels in the polymer at the fibre failure strains $\left(\sigma_{\mathrm{m}}^{\prime}\right)$ of 0.05 per cent $(\mathrm{PZT}-5 \mathrm{H})$ and 0.06 per cent (PZT$5 \mathrm{~A})$ were 1.5 and $1.8 \mathrm{MPa}$, respectively. The ultimate failure strain of the matrix $\left(\varepsilon_{\mathrm{m}}^{*}\right)$ was typically $2-3$ per cent, two orders of magnitude greater than that of the brittle fibres.

\subsection{PZT-5H/epoxy composite properties}

Using the composite theory described earlier and the appropriate material properties, composite strength as a function of volume fraction was determined from equations (2) and (3). The predicted composite strength versus fibre volume fraction $V_{\mathrm{f}}$ for a PZT$5 \mathrm{H} /$ epoxy composite is shown in Fig. 8 . The solid line is the predicted composite strength at each volume fraction and the inset shows the failure modes above and below $V_{\mathrm{min}}$. The minimum volume fraction is 62 per cent for fibre volume fractions in the range of $0<V_{\mathrm{f}}<62$ per cent, failure of the fibres does not lead to composite failure. As $V_{\mathrm{f}}$ increases in this range of fibre volume fractions, there is a gradual decrease in the composite tensile strength from the matrix value of 57 to $21 \mathrm{MPa}$ at $V_{\mathrm{f}}=62$ per cent.

For fibre fractions greater than 62 per cent, fibre failure leads to the failure of the composite (Fig. 3(c)). For a monolayer of cylindrical

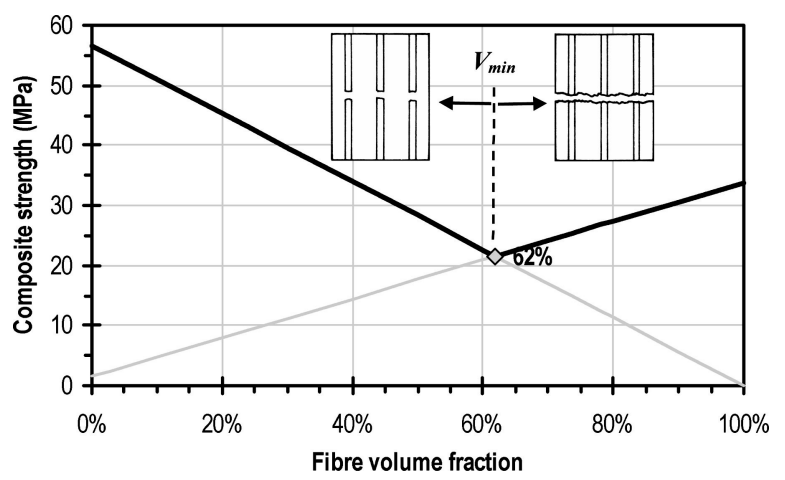

Fig. 8 Predicted strength for PZT-5H/epoxy composites with different fibre volume fractions. Parameters used: $\varepsilon_{\mathrm{f}}^{*}=0.05$ per cent, $\sigma_{\mathrm{f}}^{*}=34 \mathrm{MPa}, \sigma_{\mathrm{m}}^{*}=$ $57 \mathrm{MPa}$, and $\sigma_{\mathrm{m}}^{\prime}=1.5 \mathrm{MPa}$. Inset shows failure modes above and below $V_{\min }$ piezoelectric fibres in an epoxy matrix, as in Fig. 2, the maximum theoretical volume fraction is 78 per cent, although slightly lower fractions are desirable to minimize fibre contact and optimize stress distribution. At the maximum volume fraction of 78 per cent the tensile strength is $\sim 25 \mathrm{MPa}$. Higher volume fractions and strengths could be achievable with fibres of rectangular cross-section. As the tensile strength of the PZT fibres (34 MPa) is lower than that of the matrix ( $56 \mathrm{MPa}$ ), the composite strength is always lower than that of the matrix, and as a result there is no critical volume fraction $\left(V_{\text {crit }}\right)$ where the composite strength exceeds that of the matrix.

\subsection{PZT-5A/epoxy composite properties}

The predicted composite strength as a function of fibre volume fraction is shown in Fig. 9 for the PZT$5 \mathrm{~A}$ system. The higher strength of the PZT-5A when compared with PZT-5H leads to a lower value of $V_{\text {min }}$ of 58 per cent. For fibre volume fractions of $0<V_{\mathrm{f}}<58$ per cent, the strength of the composite decreases with increasing fibre content, to a minimum of $\sim 23 \mathrm{MPa}$. At the maximum packing density of 78 per cent, the composite strength is predicted to be $\sim 31 \mathrm{MPa}$. As the PZT-5A fibres are also weaker than the matrix, the composite strength never exceeds that of the matrix. As a result, there is no critical fibre volume fraction $\left(V_{\text {crit }}\right)$.

Recently reported mechanical properties of PZT5A fibres [24] have also been used to predict the strength variation of the PZT-5A/epoxy composites (Fig. 10). The reported higher fibre strength ( $\sim 68 \mathrm{MPa})$ leads to a $V_{\min }$ of 44 per cent. As the fibres are slightly stronger than the matrix, a critical volume fraction above which the composite strength is greater than that of the matrix can be estimated. The value of $V_{\text {crit }}$ is 93 per cent however, such a

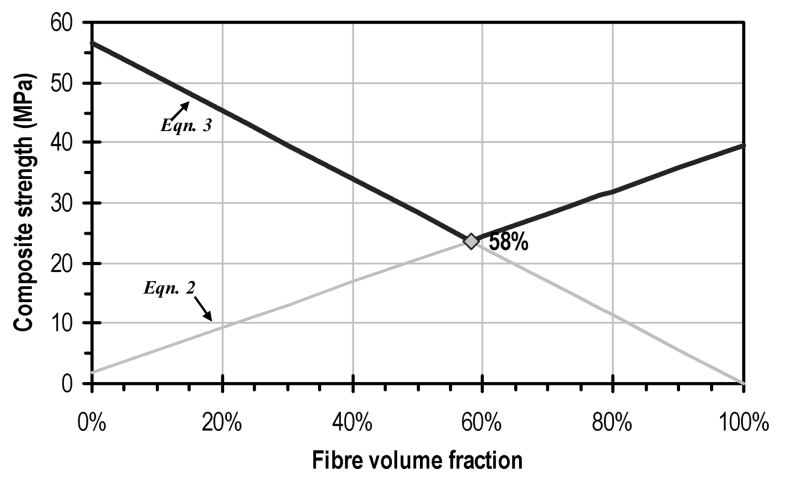

Fig. 9 Predicted strength for PZT-5A/epoxy composites with different fibre volume fractions. Parameters used: $\varepsilon_{\mathrm{f}}^{*}=0.06$ per cent, $\sigma_{\mathrm{f}}^{*}=40 \mathrm{MPa}, \sigma_{\mathrm{m}}^{*}=57 \mathrm{MPa}$ and $\sigma_{\mathrm{m}}^{\prime}=1.8 \mathrm{MPa}$ 


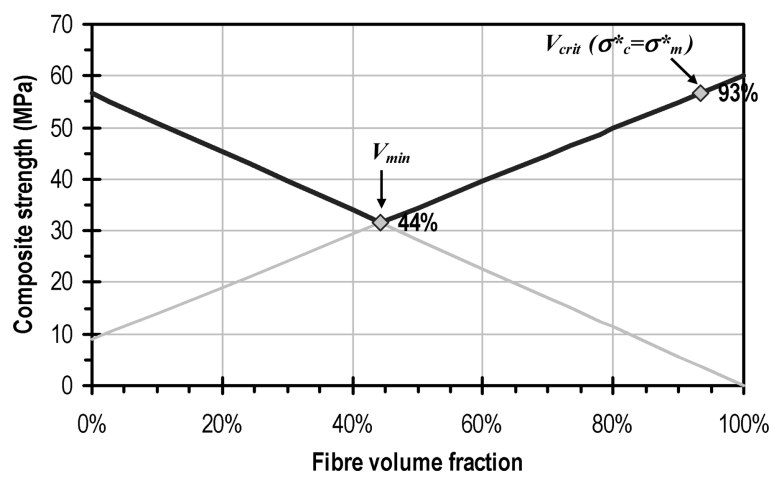

Fig. 10 Predicted strength for PZT-5A (2004)/epoxy composites with different fibre volume fractions. Parameters used: $\varepsilon_{\mathrm{f}}^{*}=0.3$ per cent, $\sigma_{\mathrm{f}}^{*}=60 \mathrm{MPa}, \sigma_{\mathrm{m}}=57 \mathrm{MPa}$, and $\sigma_{\mathrm{m}}^{*}=8.9 \mathrm{MPa}$

high volume fraction is not achievable with monolayers of cylindrical fibres, as shown in Fig. 2.

\section{DISCUSSION AND APPLICATION TO COMPOSITE DESIGN}

Piezoelectric fibre and matrix properties have been used to evaluate the tensile strength and failure mechanisms of AFCs as a function of fibre volume fraction. Two different fibre types, PZT-5H and PZT-5A each, with different failure strengths and strains, have been examined. This, therefore, represents a range of the potentially available composite tensile strengths and typical minimum volume fractions for AFC materials. The approach used has been traditionally employed to determine the tensile strengths of structural composites; however, no research to date has estimated $V_{\min }$ and $V_{\text {crit }}$ for AFCs.

For both active composite systems examined (PZT-5H/epoxy and PZT-5A/epoxy), the minimum volume fractions obtained are particularly high (44-62 per cent) when compared with engineering composites such as carbon fibre/epoxy. Critical and minimum volume fractions are typically less than 3 per cent for these structural materials, because of the much higher tensile strength of the fibre ( $>2 \mathrm{GPa}[\mathbf{1 5}]$ ) compared with the matrix. For the same reason, the AFCs also have lower tensile strengths than carbon fibre/epoxy composites.

A particular advantage of the high minimum volume fraction, $V_{\min }$, for these materials is that active fibre composites with relatively high fibre volume fractions will not fail when the individual PZT fibres fracture. If AFCs are designed to fail in this mode (as in Fig. 3(c)), they are less likely to act as a point of weakness in a host structure if they experience a high level of stress or strain. In addition, because the IDE enables the device to continue operating as a sensor or actuator after fibre fracture, this is the preferred failure mode for operation. The use of a lower fibre volume fraction is, however, likely to reduce the piezoelectric performance of the device. To quantify these effects, Fig. 11 presents PZT fibre/ epoxy composite properties for a wide range of fibre volume fractions, namely $d_{33}$ piezoelectric coefficient (strain per unit electric field) and elastic stiffness $\left(1 / s_{33}^{D}\right)$ [25]. From this work, the $V_{\text {min }}$ fractions are typically 44-60 per cent, and examination of
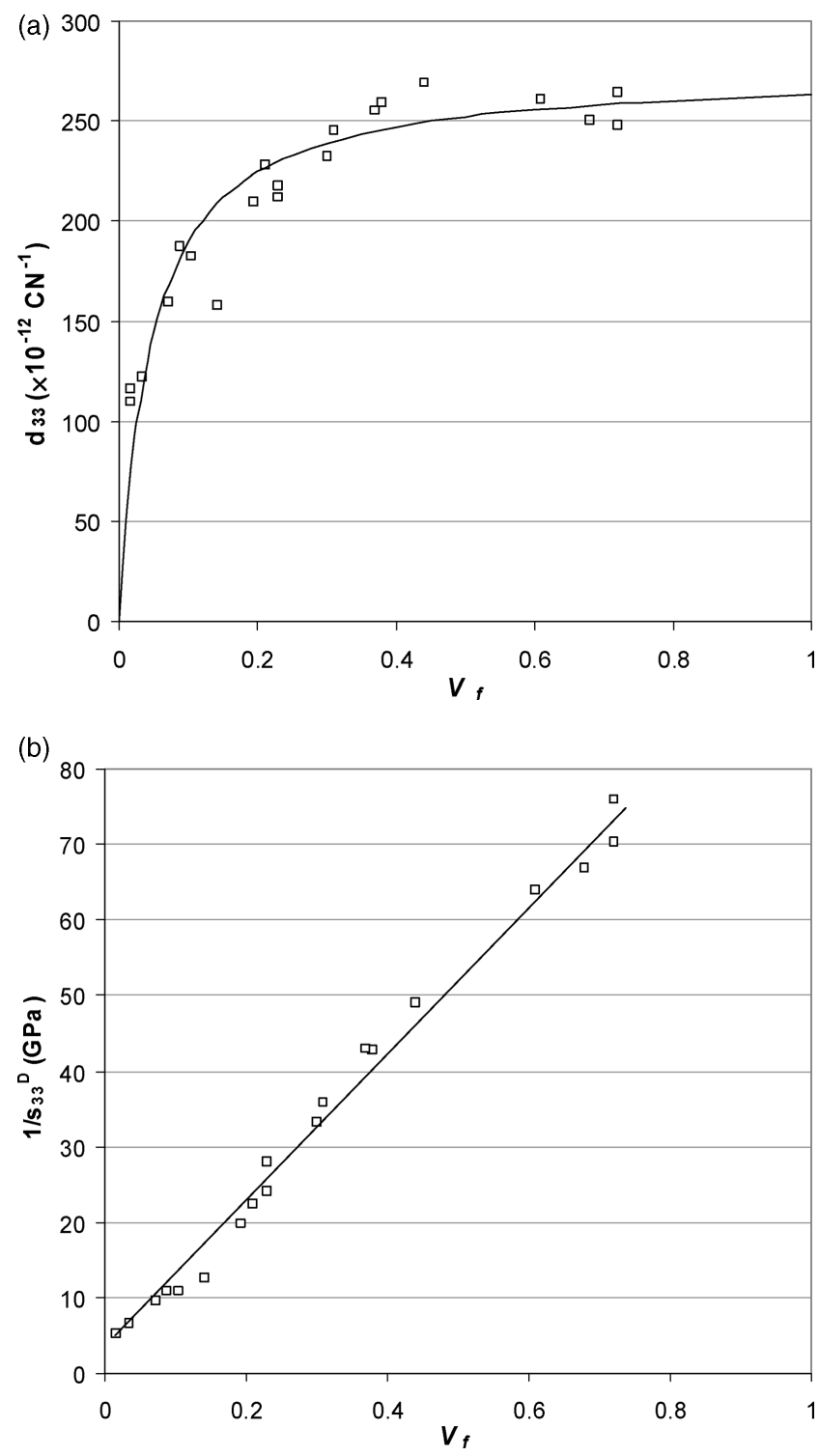

Fig. 11 Experimental results of (a) effective $d_{33}$ and (b) effective stiffness $1 / s_{33}^{D}$ of composites as a function of fibre volume fraction. The effective property data are from PZT fibre/ epoxy composites manufactured with $250 \mu \mathrm{m}$ diameter fibres. The solid lines are analytical models [25] 
Fig. 11(a) shows that the $d_{33}$ coefficient is almost unchanged in this range when compared with the fully dense material $\left(V_{\mathrm{f}}=1\right)$. This implies that by choosing a fibre volume fraction of 44-60 per cent, when compared with 78 per cent, the free piezoelectric strain is not reduced significantly. There is, however, a more significant reduction in the stiffness $\left(1 / s_{33}^{D}\right)$, as seen in Fig. 11b. The reduction in stiffness will minimize the potential force that the ACFs can produce or lead to reduced strain under combined mechanical and electrical loading.

Our analysis as presented is of particular interest for those considering the development and design of robust and damage tolerant actuators or embedding AFCs in engineering structures for structural health monitoring, vibration damping, or shape control. Further work currently being undertaken is to experimentally determine the fracture strengths of PZT fibre/epoxy composites over a range of volume fractions and to study the failure modes.

\section{ACKNOWLEDGEMENTS}

The authors acknowledge the National Physical Laboratory (NPL) and the PRIME Faraday Partnership for providing funding for this research. The $4 \mathrm{M}$ Network of Excellence is also acknowledged.

\section{REFERENCES}

1 Strock, H. B., Pascucci, M. R., Parish, M. V., Bent, A. A., and Shrout, T. R. Active PZT fibers, a commercial production process. SPIE Conf. Proc., 1999, 3675, 22-31.

2 Meyer, R., Shrout, T., and Yoshikawa, S. Lead zirconate titanate fine fibers derived from alkoxide-based sol-gel technology. J. Am. Ceram. Soc., 1998, 81, 861-868.

3 French, J. D. and Cass, R. B. Developing innovative ceramic fibers. Am. Ceram. Soc. Bull., 1998, 77, 61-65.

4 Meister, F., Vorbach, D., Niemz, F., Schulze, T., and Taeger, E. Functional high-tech-cellulose materials by the ALCER (R) process. Materialwiss Werkst., 2003, 34, 262-266.

5 Li, K., Chan, H. L. W., and Choy, C. L. Samarium and manganese-doped lead titanate ceramic fiber/epoxy 1-3 composite for high-frequency transducer application. IEEE Trans. Ultra. Ferro. Freq. Control, 2003, 50, 1371-1376.

6 Li, K., Li, J. H., Li, J. C., and Chan, H. L. W. Fabrication and properties of PLZT ceramic fiber/epoxy 1-3 composites, J. Inorg. Mater., 2004, 19, 361-366.

7 Bent, A. A. and Hagood, N. W. Piezoelectric fiber composites with interdigitated electrodes. J. Int. Mater. Syst. Struct., 1997, 8, 903-919.

8 Hagood, N. and Bent, A. A. Composites for structural control. US Pat. 6048 622, 2000.

9 Nelson, L. J. Smart piezoelectric fibre composites. Mater. Sci. Tech., 2002, 18, 1245-1256.
10 Nelson, L. J. and Bowen, C. R. Determination of the piezoelectric properties of fine scale PZT fibres. Key. Eng. Mater., 2002, 206, 1509-1512.

11 Bent, A. A., Hagood, N. W., and Rodgers, J. Anisotropic actuation with piezoelectric fibre composites. J. Intell. Mater. Syst. Struct., 1995, 6, 338-349.

12 Bent, A. A. Active fibre composites for structural actuation. $\mathrm{PhD}$ Thesis, Massachusetts Institute of Technology, 1997.

13 Schonecker, A., Keitel, U., Kreher, W., Sporn, D., Watzka, W., and Pannkoke, K. Smart structures by integrated piezoelectric thin fibres (II): properties of composites and their physical description. Ferroelectrics, 1999, 224, 435-440.

14 Moses, R. W., Wieseman, C. D., Bent, A. A., and Pizzochero, A. E. Evaluation of new actuators in a buffet loads environment. Proc. SPIE, 2001, 4332, $10-21$.

15 Harris, B. Engineering composite materials, 1986 (Institute of Materials, London).

16 Jones, R. M. Mechanics of composite materials, 2nd edition, 1999 (Taylor \& Francis, Philadelphia).

17 Nelson, L. J. Characterisation and modelling of active fibre composites. PhD Thesis, University of Bath, 2004.

18 Nelson, L. J., Bowen, C. R., Stevens, R., Cain, M., and Stewart, M. High field behaviour of piezoelectric fibre composites. Proc. SPIE, 2003, 5053, 544-555.

19 Joffe, H. and Berlincourt, D. A. Piezoelectric transducer materials. Proc. IEEE, 1965, 53, 1372-1386.

20 European Standard EN ISO 527-2:1996E. Plastics determination of tensile properties. Part 2: test conditions for moulding and extrusion plastics, 1996.

21 European Prestandard ENV1007-4:1994E. Advanced technical ceramics - ceramic composites - methods of test for reinforcements. Part 4: determination of tensile properties of filament at ambient temperature, 1994.

22 Rasband, W. S. ImageJ, 2005 (US National Institutes of Health, Bethesda, Maryland).

23 Ashby, M. F. and Jones, D. R. H. The statistics of brittle fracture In Engineering materials 2: An introduction to microstructures, processing and design, 2nd edition, 1998, pp. 178-185 (Butterworth-Heinemann, Oxford)

24 Kornmann, X. and Huber, C. Microstructure and mechanical properties of PZT fibres. J. Euro. Ceram. Soc., 2004, 24, 1987-1991.

25 Nelson, L. J., Bowen, C. R., Stevens, R., Cain, M., and Stewart, M. Modelling and measurement of piezoelectric fibres and interdigitated electrodes for the optimisation of piezofibre composites. Proc. SPIE, 2003, 5053, 556- 567

\section{APPENDIX}

\section{Notation}

$d_{33} \quad$ piezoelectric coefficient, strain per unit electric field $\left(\mathrm{mV}^{-1}\right)$

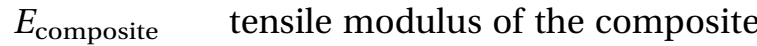
(GPa) 


$\begin{array}{llll}E_{\text {matrix }} & \text { tensile modulus of the epoxy matrix } & \varepsilon_{\mathrm{c}}^{*} & \text { composite failure strain } \\ & (\mathrm{GPa}) & \varepsilon_{\mathrm{f}}^{*} & \text { fibre failure strain } \\ E_{\mathrm{PZT}} & \text { tensile modulus of the lead zirconate } & \varepsilon_{\mathrm{m}}^{*} & \text { matrix failure strain } \\ & \text { titanate }(\mathrm{PZT}) \text { fibres (GPa) } & v & \text { Poisson's ratio } \\ m & \text { Weibull shape parameter } & \sigma_{\mathrm{c}}^{*} & \text { composite failure stress (MPa) } \\ s_{33}^{D} & \text { compliance under constant } & \sigma_{\mathrm{f}}^{*} & \text { fibre failure stress (MPa) } \\ & \text { polarization }\left(\mathrm{Pa}^{-1}\right) & \sigma_{\mathrm{m}}^{*} & \text { matrix failure stress (MPa) } \\ V_{\text {crit }} & \text { critical fibre volume fraction } & \sigma_{\mathrm{m}}^{\prime} & \text { stress in the polymer matrix at the } \\ V_{\mathrm{f}} & \text { fibre volume fraction } & & \text { fibre failure strain (MPa) } \\ V_{\mathrm{min}} & \text { minimum fibre volume fraction } & \sigma_{0} & \text { Weibull-scale parameter (MPa) }\end{array}$

